\title{
Properties of Commutativity of Dual Toeplitz Operators on the Orthogonal Complement of Pluriharmonic Dirichlet Space over the Ball
}

\author{
Yinyin Hu, Yufeng Lu, and Tao Yu \\ School of Mathematical Sciences, Dalian University of Technology, Dalian 116024, China \\ Correspondence should be addressed to Yinyin Hu; 534480549@qq.com
}

Received 23 October 2015; Accepted 24 December 2015

Academic Editor: Kehe Zhu

Copyright (C) 2016 Yinyin Hu et al. This is an open access article distributed under the Creative Commons Attribution License, which permits unrestricted use, distribution, and reproduction in any medium, provided the original work is properly cited.

We completely characterize the pluriharmonic symbols for (semi)commuting dual Toeplitz operators on the orthogonal complement of the pluriharmonic Dirichlet space in Sobolev space of the unit ball. We show that, for $f$ and $g$ pluriharmonic functions, $S_{f} S_{g}=S_{g} S_{f}$ on $\left(\mathscr{D}_{h}\right)^{\perp}$ if and only if $f$ and $g$ satisfy one of the following conditions: (1) both $f$ and $g$ are holomorphic;

(2) both $\bar{f}$ and $\bar{g}$ are holomorphic; (3) there are constants $\alpha$ and $\beta$, both not being zero, such that $\alpha f+\beta g$ is constant.

\section{Introduction}

For any integer $n>1$, let $B_{n}$ denote the open unit ball in $C^{n}$. The boundary of $B_{n}$ is the sphere $S_{n}$ and the closure of $B_{n}$ with the Euclidean metric on $C^{n}$ is denoted by $\overline{B_{n}}$. Let $d \nu$ denote the Lebesgue volume measure on the unit ball $B_{n}$ of $C^{n}$, normalized so that the measure of $B_{n}$ equals 1 . The Sobolev space $W^{1,2}=W^{1,2}\left(B_{n}, d \nu\right)$ is the completion of the collection of all smooth functions $f$ on $B_{n}$ for which

$$
\begin{aligned}
\|f\| & =\left[\left|\int_{B_{n}} f(z) d \nu(z)\right|^{2}\right. \\
& \left.+\sum_{i=1}^{n} \int_{B_{n}}\left\{\left|\frac{\partial f}{\partial z_{i}}\right|^{2}+\left|\frac{\partial f}{\partial \overline{z_{i}}}\right|^{2}\right\} d \nu(z)\right]^{1 / 2}<\infty,
\end{aligned}
$$

where $\partial / \partial z_{i}, \partial / \partial \overline{z_{i}}$ is the weak partial derivative. The $W^{1,2}\left(B_{n}, d \nu\right)$ is a Hilbert space with the inner product

$$
\begin{aligned}
\langle f, g\rangle= & \int_{B_{n}} f(z) d \nu(z) \int_{B_{n}} \overline{g(z)} d \nu(z) \\
& +\sum_{i=1}^{n}\left[\left\langle\frac{\partial f}{\partial z_{i}}, \frac{\partial g}{\partial z_{i}}\right\rangle_{2}+\left\langle\frac{\partial f}{\partial \overline{z_{i}}}, \frac{\partial g}{\partial \overline{z_{i}}}\right\rangle_{2}\right],
\end{aligned}
$$

where $\langle\cdot, \cdot\rangle_{2}$ denotes the inner product in the Lebesgue space $L^{2}\left(B_{n}, d v\right)$. The Dirichlet space $\mathscr{D}=\mathscr{D}\left(B_{n}, d v\right)$ is the closed subspace of $W^{1,2}\left(B_{n}, d \nu\right)$ consisting of all holomorphic functions, and let $P$ denote the orthogonal projection from $W^{1,2}\left(B_{n}, d \nu\right)$ onto $\mathscr{D}\left(B_{n}, d \nu\right)$. Then $P$ is an integral operator represented by

$$
P(f)(w)=\left\langle f, K_{w}\right\rangle=\int_{B_{n}} f d \nu+\sum_{i=1}^{n} \int_{B_{n}} \frac{\partial f}{\partial z_{i}} \frac{\overline{\partial K_{w}}}{\partial z_{i}} d \nu
$$

where $K_{w}(z)=K(z, w)$ is the reproducing kernel of $\mathscr{D}$. By computation, we know that

$$
K(z, w)=1+\sum_{\alpha \in \mathbb{N}^{n}-\{0\}} \frac{(|\alpha|+n-1) !}{n ! \alpha !|\alpha|} z^{\alpha} \bar{w}^{\alpha},
$$

where $\{0\}=(0, \ldots, 0), \alpha=\left(\alpha_{1}, \ldots, \alpha_{n}\right) \in \mathbb{N}^{n}, \alpha !=$ $\alpha_{1} ! \cdots \alpha_{n} !, z^{\alpha}=z_{1}^{\alpha_{1}} \cdots z_{n}^{\alpha_{n}},|\alpha|=\sum_{i=1}^{n} \alpha_{i}$ and $\mathbb{N}$ is the set of nonnegative integers. The pluriharmonic Dirichlet space $\mathscr{D}_{h}$ is the closed subspace of $W^{1,2}\left(B_{n}, d \nu\right)$ consisting of all pluriharmonic functions. Let $Q$ denote the orthogonal projection from $W^{1,2}$ onto $\mathscr{D}_{h}$; then $(Q f)(z)=\left\langle f, R_{z}\right\rangle$, where $R_{z}=K_{z}+\overline{K_{z}}-1$. In fact,

$$
(Q f)(z)=(P f)(z)+\overline{(P \bar{f})(z)}-(P f)(0) .
$$


Let

$$
\begin{aligned}
& W^{1, \infty}\left(B_{n}\right)=\left\{\varphi \in W^{1,2}\left(B_{n}, d \nu\right): \varphi, \frac{\partial \varphi}{\partial z_{i}}, \frac{\partial \varphi}{\partial \overline{z_{i}}}\right. \\
& \left.\quad \in L^{\infty}\left(B_{n}, d \nu\right), i=1,2, \ldots, n\right\} .
\end{aligned}
$$

Given a function $f \in W^{1, \infty}\left(B_{n}\right)$, the multiplication operator $M_{f}$, the Toeplitz operator $T_{f}$, the Hankel operator $H_{f}$, the dual Toeplitz operator $S_{f}$, and the dual Hankel operator $R_{f}$ with symbol $f$ are defined, respectively, by

$$
\begin{aligned}
& M_{f}: W^{1,2} \longrightarrow W^{1,2}, \quad M_{f}(h)=f h, h \in W^{1,2} ; \\
& T_{f}: \mathscr{D}_{h} \longrightarrow \mathscr{D}_{h}, \quad T_{f}(h)=Q(f h), h \in \mathscr{D}_{h} ; \\
& H_{f}: \mathscr{D}_{h} \longrightarrow \mathscr{D}_{h}^{\perp}, \\
& H_{f}(h)=(I-Q)(f h), h \in \mathscr{D}_{h} ; \\
& S_{f}: \mathscr{D}_{h}^{\perp} \longrightarrow \mathscr{D}_{h}^{\perp}, \quad S_{f}(h)=(I-Q)(f h), h \in \mathscr{D}_{h}^{\perp} ; \\
& R_{f}: \mathscr{D}_{h}^{\perp} \longrightarrow \mathscr{D}_{h}, \quad R_{f}(h)=Q(f h), h \in \mathscr{D}_{h}^{\perp} .
\end{aligned}
$$

They are all bounded linear operators. Under the decomposition $W^{1,2}=\mathscr{D}_{h} \oplus\left(\mathscr{D}_{h}\right)^{\perp}$, the multiplication operator $M_{f}$ is represented as

$$
\left(\begin{array}{cc}
T_{f} & R_{f} \\
H_{f} & S_{f}
\end{array}\right)
$$

This shows close relationships among the above four types of operators. Many studies for dual Toeplitz operators offer some insights into the study for Toeplitz operators. So it is reasonable to focus on the dual Toeplitz operators. Although dual Toeplitz operators differ in many ways from Toeplitz operators, they do have some of the same properties. The general problem that we are interested in is the following: what is the relationship between their symbols when two dual Toeplitz operators commute?

For Toeplitz operators, this problem has been studied for a long time. In the case of the classical Hardy space, Brown and Halmos [1] showed that two Toeplitz operators with general bounded symbols commute if and only if either both symbols are analytic, both symbols are conjugate analytic, or a nontrivial linear combination of the symbols is constant.

Initiated by Brown and Halmos's pioneering work, the problem of characterizing when two Toeplitz operators commute has been one of the topics of constant interest in the study of Toeplitz operators on classical function spaces over various domains. On the Bergman space of the unit disk, Axler and Čučković [2] studied commuting Toeplitz operators with harmonic symbols and obtained a similar result to that of Brown and Halmos. Stroethoff [3] later extended that result to essentially commuting Toeplitz operators. Axler et al. [4] showed that if two Toeplitz operators commute and the symbol of one of them is nonconstant analytic, then the other one must be analytic. Čučković and Rao [5] studied Toeplitz operators that commute with Toeplitz operators with monomial symbols. On the Bergman space of several complex variables, by making use of $\mathscr{M}$-harmonic function theory, Zheng [6] characterized commuting Toeplitz operators with pluriharmonic symbols on the Bergman space of the unit ball. Choe and Lee [7-9] studied commuting and essentially commuting Toeplitz operators with pluriharmonic symbols on the unit ball. Lu [10] characterized commuting Toeplitz operators on the bidisk with pluriharmonic symbols. Choe et al. [11] obtained characterizations of (essentially) commuting Toeplitz operators with pluriharmonic symbols on the Bergman space of the polydisk.

The fact that the product of two harmonic functions is no longer harmonic adds some mystery to the study of operators on harmonic Bergman space. Many methods which work for the operators on analytic Bergman space lose their effectiveness on harmonic Bergman space. On the harmonic Bergman space of the unit disk, Ohno [12] first characterized the commutativity of $T_{f}$ and $T_{z}$, where $f$ is an analytic function. Choe and Lee [13] studied commuting Toeplitz operator with harmonic symbols and one of the symbols is a polynomial. In [14], Choe and Lee proved that if $f, g \in H^{\infty}$ and supposedly one of them is noncyclic, then $T_{f} T_{\bar{g}}=T_{g} T_{\bar{f}}$ if and only if either $f$ or $g$ is constant. On the pluriharmonic Bergman space of the unit ball, commuting Toeplitz operators were studied in $[15,16]$.

However, the study on the problem for dual Toeplitz operators started recently. Stroethoff and Zheng [17] characterized the commutativity of dual Toeplitz operators with bounded symbols on the orthogonal complement of the Bergman space of the unit disk and studied algebraic and spectral properties of dual Toeplitz operators. On the Bergman space of the unit ball and the polydisk, commuting dual Toeplitz operators were studied in [18-20]. Yang and Lu [21] gave complete characterization for the (semi)commuting dual Toeplitz operators with harmonic symbols on harmonic Bergman space.

In recent years the Dirichlet space has received a lot of attention from mathematicians in the areas of modern analysis, probability, and statistical analysis. Many mathematicians are interested in function theory and operator theory on the Dirichlet space. Yu and $\mathrm{Wu}[22,23]$ investigated commuting dual Toeplitz operators with harmonic symbols on the Dirichlet space. Yu [24] obtained the commutativity of dual Toeplitz operators with general symbols on Dirichlet space.

In this paper, we want to characterize commuting dual Toeplitz operators with pluriharmonic symbols on the orthogonal complement of the pluriharmonic Dirichlet space in Sobolev space of the unit ball.

We state our main result now. We postpone the proofs of these theorems until Section 3.

Theorem 1. Suppose that $f, g \in W^{1, \infty}\left(B_{n}\right)$ are pluriharmonic functions; then $S_{f g}=S_{f} S_{g}$ if and only if one of the following statements holds:

(1) Both $f$ and $g$ are holomorphic.

(2) Both $\bar{f}$ and $\bar{g}$ are holomorphic.

(3) Either $f$ or $g$ is constant. 
Theorem 2. Suppose that $f, g \in W^{1, \infty}\left(B_{n}\right)$ are pluriharmonic functions, then $S_{g} S_{f}=S_{f} S_{g}$ if and only if one of the following statement holds:

(1) Both $f$ and $g$ are holomorphic.

(2) Both $\bar{f}$ and $\bar{g}$ are holomorphic.

(3) There are constants $\alpha$ and $\beta$, both not being zero, such that $\alpha f+\beta g$ is constant.

For $n=1$, two dual Toeplitz operators with harmonic symbols always commute on the orthogonal complement of harmonic Dirichlet space; that is, $S_{f} S_{g}=S_{g} S_{f}$ holds for all harmonic functions $f$ and $g$.

A pluriharmonic function in the unit ball is the sum of a holomorphic function and the conjugate of a holomorphic function. It is clear that all pluriharmonic functions on $B_{n}$ are $\mathscr{M}$-harmonic. A good reference for the function theory of the unit ball is Rudin's book [25].

The difficult part of the proof of Theorem 2 is to answer the following question about pluriharmonic functions.

Question. If $f_{1}, \ldots, f_{N}$ and $g_{1}, \ldots, g_{N}$ are holomorphic functions in $B_{n}$, when is $f_{1} \overline{g_{1}}+\cdots+f_{N} \overline{g_{N}}$ pluriharmonic?

This question is very subtle. If $N=2$, this question is a special case of Theorem 5.6 in [6]. In [26], Choe et al. gave a necessary and sufficient condition for this question in Lemma 4.7, which is useless to the proof of Theorem 2. In this paper, we give another characterization to the question and induce the proof of Theorem 2 .

\section{Some Lemmas}

The following Lemma has been known to be true for $n=1$ in [24]. For $n>1$, the following lemma may be known, but we cannot find its proof; for completeness, we give its proof.

Lemma 3. The set of all polynomials in $z$ and $\bar{z}$ is dense in $W^{1,2}\left(B_{n}\right)$.
Proof. We will discuss it in the case of real variables. For $u \in$ $W^{1,2}\left(B_{n}\right)$ and $z_{j}=x_{j}+y_{j}$, since $\partial u / \partial z_{j}=(1 / 2)\left(\partial u / \partial x_{j}-\right.$ $\left.i\left(\partial u / \partial y_{j}\right)\right)$ and $\partial u / \partial \overline{z_{j}}=(1 / 2)\left(\partial u / \partial x_{j}+i\left(\partial u / \partial y_{j}\right)\right)$, one can see that the norm of $u$ is equivalent to the following norm:

$$
\begin{gathered}
\|u\|_{r}=\left(\left|\frac{1}{M} \int_{B_{n}} u d x_{1} d y_{1} \cdots d x_{n} d y_{n}\right|^{2}+\frac{1}{M}\right. \\
\cdot \int_{B_{n}}\left[\left|\frac{\partial u}{\partial x_{1}}\right|^{2}+\left|\frac{\partial u}{\partial y_{1}}\right|^{2}+\cdots+\left|\frac{\partial u}{\partial x_{n}}\right|^{2}\right. \\
\left.\left.+\left|\frac{\partial u}{\partial y_{n}}\right|^{2}\right] d x_{1} d y_{1} \cdots d x_{n} d y_{n}\right)^{1 / 2}
\end{gathered}
$$

where $M=\int_{B_{n}} d x_{1} d y_{1} \cdots d x_{n} d y_{n}$. For any $f \in W^{1,2}\left(B_{n}\right)$ and $\varepsilon>0$, by Theorem 3.18 in [27], there exists a smooth function $u \in C_{0}^{\infty}\left(R^{2 n}\right)$ such that $\|f-u\|<\varepsilon$. Choose a constant $R \geq 1$ such that the support set of $u$ is contained in

$$
\begin{aligned}
K & =\left\{\left(x_{1}, y_{1}, \ldots, x_{n}, y_{n}\right):\left|x_{j}\right| \leq R,\left|y_{j}\right| \leq R \text { for } j\right. \\
& =1, \ldots, n\} .
\end{aligned}
$$

It follows that the support set of $\partial^{2 n} u / \partial x_{1} \partial y_{1} \cdots \partial x_{n} \partial y_{n}$ is also in $K$. Let $p$ be a polynomial such that

$$
\begin{aligned}
& \left|p\left(x_{1}, y_{1}, \ldots, x_{n}, y_{n}\right)-\frac{\partial^{2 n} u\left(x_{1}, y_{1}, \ldots, x_{n}, y_{n}\right)}{\partial x_{1} \partial y_{1} \cdots \partial x_{n} \partial y_{n}}\right| \\
& \quad<\frac{\epsilon}{4^{n} R^{2 n}}
\end{aligned}
$$

for all $\left(x_{1}, y_{1}, \ldots, x_{n}, y_{n}\right)$ in $K$, and let

$$
\begin{aligned}
p_{1} & \left(x_{1}, y_{1}, \ldots, x_{n}, y_{n}\right) \\
& =\int_{-R}^{y_{1}} \int_{-R}^{x_{2}} \ldots \int_{-R}^{x_{n}} \int_{-R}^{y_{n}} p\left(x_{1}, t_{2}, \ldots, t_{2 n}\right) d t_{2} \ldots d t_{2 n} .
\end{aligned}
$$

Similarly, we also can define $p_{j}$ for $j=1, \ldots, 2 n$. It is obtained that

$$
\left|p_{1}\left(x_{1}, y_{1}, \ldots, x_{n}, y_{n}\right)-\frac{\partial u}{\partial x_{1}}\right| \leq \int_{-R}^{y_{1}} \int_{-R}^{x_{2}} \cdots \int_{-R}^{x_{n}} \int_{-R}^{y_{n}}\left|\left(p-\frac{\partial^{2 n} u}{\partial x_{1} \partial y_{1} \cdots \partial x_{n} \partial y_{n}}\right)\left(x_{1}, t_{2}, \ldots, t_{2 n}\right)\right| d t_{2} \cdots d t_{2 n} \leq \frac{\varepsilon}{2 R}
$$

Similarly, we have $\left|p_{2 j-1}-\partial u / \partial x_{j}\right| \leq \varepsilon / 2 R$ and $\left|p_{2 j}-\partial u / \partial y_{j}\right| \leq$ $\varepsilon / 2 R$ for any $j=1, \ldots, n$.

Let $q$ denote the polynomial $\int_{-R}^{x_{1}} p_{1}\left(t, y_{1}, \ldots, x_{n}, y_{n}\right) d t$. Also we have

$$
\begin{gathered}
\int_{-R}^{x_{1}} p_{1}\left(t, y_{1}, \ldots, x_{n}, y_{n}\right) d t=\cdots \\
=\int_{-R}^{y_{n}} p_{2 n}\left(x_{1}, y_{1}, \ldots, x_{n}, t\right) d t .
\end{gathered}
$$

Similar to the above one can see that

$$
\left|q\left(x_{1}, y_{1}, \ldots, x_{n}, y_{n}\right)-u\left(x_{1}, y_{1}, \ldots, x_{n}, y_{n}\right)\right|<\varepsilon
$$

for all $\left(x_{1}, y_{1}, \ldots, x_{n}, y_{n}\right)$ in $K$. Thus we have $\|q-u\|_{r}<$ $\sqrt{2 n+1} \varepsilon$. This completes the proof. 
For two multi-indexes $\alpha=\left(\alpha_{1}, \ldots, \alpha_{n}\right)$ and $\beta=$ $\left(\beta_{1}, \ldots, \beta_{n}\right)$, the notation $\alpha>\beta$ means that

$$
\begin{aligned}
& \alpha \neq \beta, \\
& \alpha_{i} \geq \beta_{i}, \quad i=1, \ldots, n .
\end{aligned}
$$

The standard orhonormal basis for $\mathbb{C}^{n}$ consists of the vectors $d_{1}, d_{2}, \ldots, d_{n}$, where $d_{k}$ is the ordered $n$-tuple that has 1 in the $k$ th spot and 0 everywhere else. A direct computation gives that

$$
\begin{aligned}
& Q\left(z^{\alpha} \bar{z}^{\beta}\right) \\
& \quad= \begin{cases}\frac{\alpha !}{(\alpha-\beta) !} \frac{(n+|\alpha|-|\beta|-1) !}{(n+|\alpha|-1) !} z^{\alpha-\beta}, & \alpha>\beta ; \\
\frac{n ! \alpha !}{(n+|\alpha|) !}, & \alpha=\beta ; \\
\frac{\beta !}{(\beta-\alpha) !} \frac{(n+|\beta|-|\alpha|-1) !}{(n+|\beta|-1) !} \bar{z}^{\beta-\alpha}, & \alpha<\beta ; \\
0, & \text { else. }\end{cases}
\end{aligned}
$$

Let $\mathcal{N}=\operatorname{span}\left\{z^{\alpha} \bar{z}^{\beta}-Q\left(z^{\alpha} \bar{z}^{\beta}\right): \alpha, \beta \geq 0\right\}$ and we have the following Lemma.

Lemma 4. Set $\mathcal{N}$ is dense in $\mathscr{D}_{h}^{\perp}$.

Proof. Since polynomials are dense in $W^{1,2}$ by Lemma 3 and $I-Q$ is a bounded operator, we get that $N$ is dense in $\mathscr{D}_{h}^{\perp}$.

The following lemma will be useful for the proof of the main theorem.

Lemma 5. Suppose that $f \in W^{1, \infty}\left(B_{n}\right)$ is holomorphic; then we have $R_{f}\left(\mathscr{D}_{h}^{\perp}\right) \subset \mathscr{D}, R_{\bar{f}}\left(\mathscr{D}_{h}^{\perp}\right) \subset \overline{\mathscr{D}}$.

Proof. Since $\mathcal{N}$ is dense in $\left(\mathscr{D}_{h}\right)^{\perp}$, it suffices to prove $R_{f}\left[z^{\alpha} \bar{z}^{\beta}-Q\left(z^{\alpha} \bar{z}^{\beta}\right)\right] \in \mathscr{D}$ for $\alpha, \beta \in \mathbb{N}^{n}-\{0\}$. Since $f \in W^{1, \infty}$ is holomorphic, we have $f=\sum_{m \geq 0} a_{m} z^{m}$. For $\alpha=\beta$, it follows that

$$
\begin{aligned}
R_{f} & {\left[z^{\alpha} \bar{z}^{\alpha}-Q\left(z^{\alpha} \bar{z}^{\alpha}\right)\right]=R_{f}\left[z^{\alpha} \bar{z}^{\alpha}-\frac{n ! \alpha !}{(n+|\alpha|) !}\right] } \\
& =Q\left[\sum_{m \geq 0} a_{m} z^{m+\alpha} \bar{z}^{\alpha}-\frac{n ! \alpha !}{(n+|\alpha|) !} \sum_{m \geq 0} a_{m} z^{m}\right] \\
& =\sum_{m \geq 0} a_{m}\left[\frac{(m+\alpha) !}{m !} \frac{(n+|m|-1) !}{(n+|m|+|\alpha|-1) !}\right. \\
& \left.-\frac{n ! \alpha !}{(n+|\alpha|) !}\right] z^{m}
\end{aligned}
$$

in the Dirichlet space. For $\alpha>\beta$, a direct computation gives that

$$
R_{f}\left[z^{\alpha} \bar{z}^{\beta}-\frac{\alpha !}{(\alpha-\beta) !} \frac{(n+|\alpha|-|\beta|-1) !}{(n+|\alpha|-1) !} z^{\alpha-\beta}\right]
$$

$$
\begin{aligned}
& =\sum_{m \geq 0} a_{m}\left[\frac{(\alpha+m) !(n+|\alpha|+|m|-|\beta|-1) !}{(\alpha+m-\beta) !(n+|\alpha|+|m|-1) !}\right. \\
& \left.-\frac{\alpha !(n+|\alpha|-|\beta|-1) !}{(\alpha-\beta) !(n+|\alpha|-1) !}\right] z^{\alpha+m-\beta}
\end{aligned}
$$

which is also in $\mathscr{D}$. For $\alpha<\beta$, it is obtained that

$$
\begin{aligned}
R_{f} & {\left[z^{\alpha} \bar{z}^{\beta}-\frac{\beta !}{(\beta-\alpha) !} \frac{(n+|\beta|-|\alpha|-1) !}{(n+|\beta|-1) !} \bar{z}^{\beta-\alpha}\right] } \\
& =Q\left[\sum_{m \geq 0} a_{m} z^{\alpha+m} \bar{z}^{\beta}\right. \\
& \left.-\frac{\beta !}{(\beta-\alpha) !} \frac{(n+|\beta|-|\alpha|-1) !}{(n+|\beta|-1) !} \sum_{m \geq 0} a_{m} z^{m} \bar{z}^{\beta-\alpha}\right] \\
& =-a_{\beta-\alpha} \frac{n ! \beta !}{(n+|\beta|) !} \frac{|\alpha|}{(n+|\beta|-|\alpha|)} \\
& +\sum_{m>\beta-\alpha} c(m, \beta, \alpha) a_{m} z^{m+\alpha-\beta},
\end{aligned}
$$

where $c(m, \beta, \alpha)=(m+\alpha) !(n+|m|+|\alpha|-|\beta|-1) ! /(m+\alpha-$ $\beta) !(n+|\alpha|+|m|-1) !-\beta ! /(\beta-\alpha) !((n+|\beta|-|\alpha|-1) ! /(n+|\beta|-$ $1) !)(m !(n+|m|+|\alpha|-|\beta|-1) ! /(m+\alpha-\beta) !(n+|m|-1) !)$.

The last case is similar; we omit the proof. Hence we get that if $f \in W^{1, \infty}$ and $f$ is holomorphic, we have $R_{f}\left(\left(\mathscr{D}_{h}\right)^{\perp}\right) \subset$ $\mathscr{D}$. As the same discussion, we can deduce that $R_{\bar{f}}\left(\left(\mathscr{D}_{h}\right)^{\perp}\right) \subset$ $\overline{\mathscr{D}}$.

In the following proposition, we give an answer to the question that when a dual Toeplitz operator equals zero.

Proposition 6. Suppose that $f \in W^{1, \infty}$ is a pluriharmonic function. Then $S_{f}=0$ if and only if $f \equiv 0$.

Proof. Assume that $S_{f}=0$. Let

$$
h_{1}=z^{d_{1}} \bar{z}^{d_{1}}+\cdots+z^{d_{n}} \bar{z}^{d_{n}}-\frac{n}{n+1} \in\left(\mathscr{D}_{h}\right)^{\perp} .
$$

A direct computation gives that

$$
\begin{aligned}
\left(S_{f} h_{1}\right)(z) & =(I-Q)\left(f h_{1}\right)(z) \\
& =f(z)\left(|z|^{2}-\frac{n}{n+1}\right)-\frac{1}{n+1} f(z) \\
& =f(z)\left(|z|^{2}-1\right)=0 .
\end{aligned}
$$

Since $|z|<1$, it follows that $f \equiv 0$. The converse part is easy to see.

If $f, g, h$, and $k$ are holomorphic functions in $B_{n}$, when is $f \bar{g}-h \bar{k} \mathscr{M}$-harmonic? In [6], Zheng gives a necessary and sufficient condition for this question. In the following lemma, we give a generalization. For $z, w \in C^{n}$, the inner product of $z$ and $w$ is defined by $\langle z, w\rangle_{C^{n}}=\sum_{j=1}^{n} z_{j} \overline{w_{j}}$. 
Lemma 7. Suppose that $f_{1}, \ldots, f_{N}$ and $g_{1}, \ldots, g_{N}$ are holomorphic functions. Then $f_{1} \overline{g_{1}}+\cdots+f_{N} \overline{g_{N}}$ is pluriharmonic if and only if there is $N \times N$ unitary matrix:

$$
U=\left(\begin{array}{ccc}
\overline{u_{11}} & \cdots & \overline{u_{1 N}} \\
\vdots & \ddots & \vdots \\
\overline{u_{N 1}} & \cdots & \overline{u_{N N}}
\end{array}\right)=\left(\begin{array}{c}
\overline{u_{1}} \\
\vdots \\
\overline{u_{N}}
\end{array}\right)
$$

and some $1 \leq k \leq N+1$ such that $\left\langle\left(f_{1}, \ldots, f_{N}\right), u_{j}\right\rangle_{C^{N}}$ are constants for $1 \leq j \leq k-1$, and $\left\langle\left(g_{1}, \ldots, g_{N}\right), u_{j}\right\rangle_{C^{N}}$ are constants for $k \leq j \leq N$.

Proof. To prove the sufficient part, suppose that $U$ is the above unitary matrix $U=\left(\overline{u_{1}}, \ldots, \overline{u_{N}}\right)^{\top}$ such that for some $1 \leq$ $k \leq N+1,\left\langle\left(f_{1}, \ldots, f_{N}\right), u_{j}\right\rangle_{C^{N}}=c_{j}$ for $1 \leq j \leq k-1$ and $\left\langle\left(g_{1}, \ldots, g_{N}\right), u_{j}\right\rangle_{C^{N}}=c_{j}$ for $k \leq j \leq N$, where $c_{i}$ are constants. Let $f=\left(f_{1}, \ldots, f_{N}\right)$ and $g=\left(g_{1}, \ldots, g_{N}\right)$. It follows that

$$
\begin{aligned}
f_{1} \overline{g_{1}} & +\cdots+f_{N} \overline{g_{N}}=\langle f, g\rangle_{C^{N}}=\langle U f, U g\rangle_{C^{N}} \\
& =\left\langle\left(c_{1}, \ldots, c_{k-1},\left\langle f, u_{k}\right\rangle_{C^{N}}, \ldots,\left\langle f, u_{N}\right\rangle_{C^{N}}\right),\right. \\
& \left.\left(\left\langle g, u_{1}\right\rangle_{C^{N}}, \ldots,\left\langle g, u_{k-1}\right\rangle_{C^{N}}, c_{k}, \ldots, c_{N}\right)\right\rangle_{C^{N}} \\
& =\sum_{j=1}^{k-1} c_{j} \overline{\left\langle g, u_{j}\right\rangle_{C^{N}}}+\sum_{j=k}^{N} \overline{c_{j}}\left\langle f, u_{j}\right\rangle_{C^{N}} .
\end{aligned}
$$

It is obtained that $f_{1} \overline{g_{1}}+\cdots+f_{N} \overline{g_{N}}$ is pluriharmonic.

Conversely, assume that $f_{1} \overline{g_{1}}+\cdots+f_{N} \overline{g_{N}}$ is pluriharmonic. There exist two holomorphic functions $h_{1}$ and $h_{2}$ on $B_{n}$ such that

$$
f_{1} \overline{g_{1}}+\cdots+f_{N} \overline{g_{N}}+h_{1}+\overline{h_{2}}=0 .
$$

By complexifying (25) (see Lemma 2 in [28]), for all $z$ and $w$ in $B_{n}$, we get

$$
\begin{aligned}
& f_{1}(z) \overline{g_{1}(w)}+\cdots+f_{N}(z) \overline{g_{N}(w)}+h_{1}(z)+\overline{h_{2}(w)} \\
& \quad=0 .
\end{aligned}
$$

It follows that for $z$ and $w$ in $B_{n}$, we have

$$
\begin{aligned}
& \left(f_{1}(z), \ldots, f_{N}(z), h_{1}(z), 1\right) \\
& \quad \perp\left(g_{1}(w), \ldots, g_{N}(w), 1, h_{2}(w)\right) .
\end{aligned}
$$

Then there is an orthonormal basis $e_{1}, \ldots, e_{N+2}$ of $C^{N+2}$ such that for some $0 \leq k \leq N+1$,

$$
\begin{array}{rl}
\left\langle\left(f_{1}(z), \ldots, f_{N}(z), h_{1}(z), 1\right), e_{i}\right\rangle_{C^{N+2}}= & , \\
1 & 1 \leq i \leq k ; \\
\left\langle\left(g_{1}(z), \ldots, g_{N}(z), 1, h_{2}(z)\right), e_{j}\right\rangle_{C^{N+2}}= & 0, \\
k+1 & \leq j \leq N+2
\end{array}
$$

for all $z \in B_{n}$. By Gauss elimination, we eliminate $h_{1}$ and $h_{2}$. Then we get the following equations:

$$
\begin{array}{ll}
\left\langle\left(f_{1}(z), \ldots, f_{N}(z)\right), u_{i}^{\prime}\right\rangle_{C^{N}}=c_{i}^{\prime}, & 1 \leq i \leq k-1 ; \\
\left\langle\left(g_{1}(z), \ldots, g_{N}(z)\right), u_{j}^{\prime}\right\rangle_{C^{N}}=c_{j}^{\prime}, \quad k \leq j \leq N .
\end{array}
$$

Since $\left(u_{i}^{\prime}, 0, c_{i}^{\prime}\right)$ are orthogonal to $\left(u_{j}^{\prime}, c_{j}^{\prime}, 0\right)$ for $1 \leq i \leq k-1$, $k \leq j \leq N$, it follows that $u_{i}^{\prime}$ and $u_{j}^{\prime}$ are orthogonal for $1 \leq i \leq$ $k-1$ and $k \leq j \leq N$. Also the rank of the matrix $\left(\begin{array}{c}u_{1}^{\prime}, c_{1}^{\prime} \\ \vdots \\ u_{k-1}^{\prime}, c_{k-1}^{\prime}\end{array}\right)$ is $k-1$. Since the equations

$$
\left\langle f, u_{i}^{\prime}\right\rangle_{C^{N}}=c_{i}^{\prime}, \quad 1 \leq i \leq k-1
$$

have solutions, it follows that the rank of $\left\{u_{1}^{\prime}, \ldots, u_{k-1}^{\prime}\right\}$ equals $k-1$. After orthonormalization, we get orthonormal bases $u_{1}, \ldots, u_{k-1}$ such that $\left\langle f, u_{i}\right\rangle=c_{i}$ for $1 \leq i \leq k-1$. The case of $\left\{u_{k}, \ldots, u_{N}\right\}$ is similar. Then we get $N \times N$ unitary matrix $U=\left(\begin{array}{c}\overline{u_{1}} \\ \vdots \\ \overline{u_{N}}\end{array}\right)$ satisfying the lemma.

\section{Proofs of Main Theorems}

In this section, we will present the proofs of the main results.

Proof of Theorem 1. If (1) holds, we have the fact that $R_{g}\left(\mathscr{D}_{h}^{\perp}\right)$ is contained in $H\left(B_{n}\right)$. It follows that $H_{f} R_{g}=0$. The desired result follows from the equation $S_{f g}=H_{f} R_{g}+S_{f} S_{g}$. Case (2) is similar. Case (3) is easy to get the desired result.

To prove the necessity, suppose that $S_{f g}=S_{f} S_{g}$. Then we have $H_{f} R_{g}=0$. Since $f$ and $g$ are pluriharmonic functions, there exist holomorphic functions $f_{1}, f_{2}, g_{1}, g_{2}$ such that $f=$ $f_{1}+\overline{f_{2}}, g=g_{1}+\overline{g_{2}}$. Without loss of generality, we assume that $f(0)=g(0)=0$. And $g_{1}=\sum_{\alpha>0} a_{\alpha} z^{\alpha}, g_{2}=\sum_{\beta>0} b_{\beta} z^{\beta}$. Let

$$
h_{1}=z^{d_{1}} \bar{z}^{d_{1}}+\cdots+z^{d_{n}} \bar{z}^{d_{n}}-\frac{n}{n+1} \in\left(\mathscr{D}_{h}\right)^{\perp} .
$$

By a direct calculation, we have

$$
\begin{aligned}
& Q\left(g_{1} h_{1}\right)=Q\left[\sum _ { \alpha > 0 } a _ { \alpha } \left(z^{\alpha+d_{1}} z^{d_{1}}+\cdots+z^{\alpha+d_{n}} z^{d_{n}}\right.\right. \\
& \left.\left.-\frac{n}{n+1} z^{\alpha}\right)\right]=\sum_{\alpha>0} a_{\alpha} z^{\alpha}\left[\frac{\left(\alpha+d_{1}\right) !(n+|\alpha|-1) !}{\alpha !(n+|\alpha|) !}\right. \\
& \left.+\cdots+\frac{\left(\alpha+d_{n}\right) !(n+|\alpha|-1) !}{\alpha !(n+|\alpha|) !}-\frac{n}{n+1}\right]=\frac{1}{n+1} \\
& . \sum_{\alpha>0} a_{\alpha} z^{\alpha}=\frac{1}{n+1} g_{1} .
\end{aligned}
$$

Similarly, we have $Q\left(\overline{g_{2}} h_{1}\right)=(1 /(n+1)) \overline{g_{2}}$. Since $H_{f} R_{g} h_{1}=0$, it follows

$$
\frac{1}{n+1}(I-Q)\left[\left(f_{1}+\overline{f_{2}}\right)\left(g_{1}+\overline{g_{2}}\right)\right]=0 .
$$


Hence $f_{1} \overline{g_{2}}+g_{1} \overline{f_{2}} \in \mathscr{D}_{h}$ is obtained. By Theorem 5.6 in [6], we have $f_{1} \overline{g_{2}}+g_{1} \overline{f_{2}} \in \mathscr{D}_{h}$ implying that one of the following statements holds:

(1) Both $f$ and $g$ are holomorphic.

(2) Both $\bar{f}$ and $\bar{g}$ are holomorphic.

(3) Either $f$ or $g$ is constant.

(4) There is a nonzero constant $t_{1}$ such that $f_{1}-t_{1} g_{1}$ and $f_{2}+\overline{t_{1}} g_{2}$ are constants.

Then it suffices to prove that $t_{1}=0$ in condition (4) when both $g_{1}$ and $g_{2}$ are not constants. For fixed $1 \leq j \leq n$, let

$$
\begin{aligned}
& h_{2}=z^{d_{j}} \bar{z}^{d_{j}}-\frac{1}{n+1}, \\
& h_{3}=z^{2 d_{j}} \bar{z}^{2 d_{j}}-\frac{2}{(n+1)(n+2)} \in \mathscr{D}_{h}^{\perp} .
\end{aligned}
$$

In the same way, we get the following:

$$
\begin{aligned}
& f_{1} \sum_{\beta>0} b_{\beta} \frac{\beta_{j}+1}{n+|\beta|} \bar{z}^{\beta}+\overline{f_{2}} \sum_{\alpha>0} a_{\alpha} \frac{\alpha_{j}+1}{n+|\alpha|} z^{\alpha} \in \mathscr{D}_{h}, \\
& f_{1} \sum_{\beta>0} b_{\beta} \frac{\left(\beta_{j}+1\right)\left(\beta_{j}+2\right)}{(n+|\beta|)(n+|\beta|+1)} \bar{z}^{\beta} \\
& \quad+\bar{f}_{2} \sum_{\alpha>0} a_{\alpha} \frac{\left(\alpha_{j}+1\right)\left(\alpha_{j}+2\right)}{(n+|\alpha|)(n+|\alpha|+1)} z^{\alpha} \in \mathscr{D}_{h} .
\end{aligned}
$$

Applying Theorem 5.6 in [6] again, there exist two constants $t_{2}, t_{3}$ such that

$$
\begin{aligned}
& f_{1}=t_{2} \sum_{\alpha>0} a_{\alpha} \frac{\alpha_{j}+1}{n+|\alpha|} z^{\alpha}, \\
& f_{1}=t_{3} \sum_{\alpha>0} a_{\alpha} \frac{\left(\alpha_{j}+1\right)\left(\alpha_{j}+2\right)}{(n+|\alpha|)(n+|\alpha|+1)} z^{\alpha} .
\end{aligned}
$$

Therefore we have

$$
t_{1} a_{\alpha}=t_{2} a_{\alpha} \frac{\alpha_{j}+1}{n+|\alpha|}=t_{3} a_{\alpha} \frac{\left(\alpha_{j}+1\right)\left(\alpha_{j}+2\right)}{(n+|\alpha|)(n+|\alpha|+1)}
$$

for all $\alpha \in \mathbb{N}^{n}-\{0\}$ and $1 \leq j \leq n$.

Case 1. If there exist two multi-indexes $\alpha \neq \beta$ such that $a_{\alpha} \neq$ $0, a_{\beta} \neq 0$. Then from (37) we have

$$
\begin{aligned}
& t_{1}=t_{2} \frac{\alpha_{j}+1}{n+|\alpha|}=t_{3} \frac{\left(\alpha_{j}+1\right)\left(\alpha_{j}+2\right)}{(n+|\alpha|)(n+|\alpha|+1)}, \\
& t_{1}=t_{2} \frac{\beta_{j}+1}{n+|\beta|}=t_{3} \frac{\left(\beta_{j}+1\right)\left(\beta_{j}+2\right)}{(n+|\beta|)(n+|\beta|+1)} .
\end{aligned}
$$

If $t_{1} \neq 0$, then

$$
\begin{gathered}
\frac{\alpha_{j}+1}{n+|\alpha|}=\frac{\beta_{j}+1}{n+|\beta|}, \\
\frac{\left(\alpha_{j}+1\right)\left(\alpha_{j}+2\right)}{(n+|\alpha|)(n+|\alpha|+1)}=\frac{\left(\beta_{j}+1\right)\left(\beta_{j}+2\right)}{(n+|\beta|)(n+|\beta|+1)},
\end{gathered}
$$

which induces $\alpha_{j}=\beta_{j}$ for all $1 \leq j \leq n$. That is a contradiction.

Case 2. If $g_{1}$ is a monomial $a_{m} z^{m}$ for some $m \in \mathbb{N}^{n}-\{0\}$ with $a_{m} \neq 0$. Suppose that $\alpha \neq \beta$ such that

$$
\begin{aligned}
& {\left[\frac{m_{1}+\alpha_{1}+1}{n+|m|+|\alpha|}-\frac{\alpha_{1}+1}{n+|\alpha|}\right] \neq 0,} \\
& {\left[\frac{m_{1}+\beta_{1}+1}{n+|m|+|\beta|}-\frac{\beta_{1}+1}{n+|\beta|}\right] \neq 0 .}
\end{aligned}
$$

Let

$$
\begin{aligned}
& h_{4}=z^{\alpha+d_{1}} \bar{z}^{d_{1}}-\frac{\alpha_{1}+1}{n+|\alpha|} z^{\alpha}, \\
& h_{5}=z^{\beta+d_{1}} \bar{z}^{d_{1}}-\frac{\beta_{1}+1}{n+|\beta|} z^{\beta} ;
\end{aligned}
$$

a direct calculation in the same way above gives

$$
\begin{aligned}
& f_{1}=t_{4} a_{m}\left[\frac{m_{1}+\alpha_{1}+1}{n+|m|+|\alpha|}-\frac{\alpha_{1}+1}{n+|\alpha|}\right] z^{\alpha+m}, \\
& f_{1}=t_{5} a_{m}\left[\frac{m_{1}+\beta_{1}+1}{n+|m|+|\beta|}-\frac{\beta_{1}+1}{n+|\beta|}\right] z^{m+\beta} .
\end{aligned}
$$

It follows that $t_{4}=t_{5}=0$. Then the fact that $f_{1}-t_{1} g_{1}=-t_{1} g_{1}$ is a constant implies that $t_{1}=0$ which is a contradiction. Hence we get the desired result.

Suppose that $f, g$ are pluriharmonic functions and $f=$ $f_{1}+\overline{f_{2}}, g=g_{1}+\overline{g_{2}}$ where $f_{1}, f_{2}, g_{1}, g_{2}$ are holomorphic functions. We are ready to prove Theorem 2.

Proof of Theorem 2. From the equation $S_{f g}=H_{f} R_{g}+S_{f} S_{g}$, it follows that

$$
S_{f} S_{g}-S_{g} S_{f}=H_{g} R_{f}-H_{f} R_{g} .
$$

Then $S_{f} S_{g}=S_{g} S_{f}$ if and only if $H_{g} R_{f}=H_{f} R_{g}$.

Assume that $S_{f} S_{g}=S_{g} S_{f}$. Then for any $v \in \mathscr{D}_{h}^{\perp}$, we have $H_{g} R_{f} v=H_{f} R_{g} v$. It is obtained that

$$
\begin{aligned}
& (I-Q)\left[\left(f_{1}+\overline{f_{2}}\right) Q\left(g_{1} v+\overline{g_{2}} v\right)\right] \\
& \quad=(I-Q)\left[\left(g_{1}+\overline{g_{2}}\right) Q\left(f_{1} v+\overline{f_{2}} v\right)\right] .
\end{aligned}
$$

By Lemma 5, we have the fact that $Q\left(g_{1} v\right), Q\left(f_{1} v\right)$ are holomorphic and $\overline{Q\left(\overline{g_{2}} v\right)}, \overline{Q\left(\overline{f_{2}} v\right)}$ are holomorphic. Then we get

$$
\begin{aligned}
& (I-Q)\left[f_{1} Q\left(g_{1} v\right)+\overline{f_{2}} Q\left(\overline{g_{2}} v\right)\right]=0, \\
& (I-Q)\left[g_{1} Q\left(f_{1} v\right)+\overline{g_{2}} Q\left(\overline{f_{2}} v\right)\right]=0 .
\end{aligned}
$$


It follows that

$$
\begin{aligned}
& (I-Q)\left[f_{1} Q\left(\overline{g_{2}} v\right)+\overline{f_{2}} Q\left(g_{1} v\right)-g_{1} Q\left(\overline{f_{2}} v\right)\right. \\
& \left.-\overline{g_{2}} Q\left(f_{1} v\right)\right]=0 .
\end{aligned}
$$

If one of $f_{1}, g_{1}, \overline{f_{2}}, \overline{g_{2}}$ is a constant function, without loss of generality, assume that $f_{1}$ is a constant function; this follows for any $v \in\left(\mathscr{D}_{h}\right)^{\perp}$; we get

$$
(I-Q)\left[\overline{f_{2}} Q\left(g_{1} v\right)-g_{1} Q\left(\overline{f_{2}} v\right)\right]=0 .
$$

We have the fact that $\overline{f_{2}} Q\left(g_{1} v\right)-g_{1} Q\left(\overline{f_{2}} v\right)$ is pluriharmonic for all $v \in\left(\mathscr{D}_{h}\right)^{\perp}$. By Theorem 5.6 in [6], one of the following holds:

(1) Both $g_{1}$ and $\overline{f_{2}}$ are constants.

(2) Both $g_{1}$ and $Q\left(g_{1} v\right)$ are constants.

(3) Both $Q\left(\overline{f_{2}} v\right)$ and $\overline{f_{2}}$ are constants.

(4) Both $Q\left(\overline{f_{2}} v\right)$ and $Q\left(g_{1} v\right)$ are constants.

(5) There is a nonzero constant $t$ such that $g_{1}-t Q\left(g_{1} v\right)$ and $\overline{f_{2}}-t Q\left(\overline{f_{2}} v\right)$ are constants.

If $g_{1}$ is a constant function, we have the fact that both $\bar{f}$ and $\bar{g}$ are holomorphic. If $\overline{f_{2}}$ is a constant function, then $f$ is a constant function. Assume that neither $\overline{f_{2}}$ nor $g_{1}$ is constant. Then for all $v \in\left(\mathscr{D}_{h}\right)^{\perp}, \overline{f_{2}} Q\left(g_{1} v\right)-g_{1} Q\left(\overline{f_{2}} v\right)$ is pluriharmonic if and only if one of the following holds:

(1) Both $Q\left(\overline{f_{2}} v\right)$ and $Q\left(g_{1} v\right)$ are constants.

(2) There is a nonzero constant $t$ such that $g_{1}-t Q\left(g_{1} v\right)$ and $\overline{f_{2}}-t Q\left(\overline{f_{2}} v\right)$ are constants.

Since $g_{1}$ is holomorphic, $g_{1}=\sum_{m \geq 0} a_{m} z^{m}$. And $g_{1}$ is not a constant; there exists a multi-index $\beta>0$ such that $a_{\beta} \neq 0$. For any multi-index $\alpha>\beta$, let $v_{\alpha}=z^{\alpha+d_{1}} \bar{z}^{d_{1}}-\left(\left(\alpha_{1}+1\right) /(n+\right.$ $|\alpha|)) z^{\alpha} \in\left(\mathscr{D}_{h}\right)^{\perp}$. A direct computation gives

$$
Q\left(g_{1} v_{\alpha}\right)=z^{\alpha} \sum_{m \geq 0} a_{m}\left[\frac{m_{1}+\alpha_{1}+1}{n+|m|+|\alpha|}-\frac{\alpha_{1}+1}{n+|\alpha|}\right] z^{m} \text {. }
$$

We choose a $\alpha^{\prime}>\beta$ such that $\left(\left(\beta_{1}+\alpha_{1}^{\prime}+1\right) /(n+|\beta|+\right.$ $\left.\left.\left|\alpha^{\prime}\right|\right)\right)-\left(\left(\alpha_{1}^{\prime}+1\right) /\left(n+\left|\alpha^{\prime}\right|\right)\right) \neq 0$. Since $a_{\beta} \neq 0$, it follows that $Q\left(g_{1} v_{\alpha^{\prime}}\right)$ is not a constant. Then we get that there is a nonzero constant $t$ such that $g_{1}-t Q\left(g_{1} v_{\alpha^{\prime}}\right)$ is constant. Since $\alpha^{\prime}>\beta$, from the fact that $g_{1}-t Q\left(g_{1} v_{\alpha^{\prime}}\right)$ is constant, we get $a_{\beta}=0$, which is a contradiction. Hence if $f_{1}$ is a constant function, we have either both $\bar{f}$ and $\bar{g}$ are holomorphic or $f$ is a constant function.

In the following proof, assume that none of $f_{1}, g_{1}, \overline{f_{2}}$, and $\overline{g_{2}}$ is a constant function. It follows that $f_{1} Q\left(\overline{g_{2}} v\right)+\overline{f_{2}} Q\left(g_{1} v\right)-g_{1} Q\left(\overline{f_{2}} v\right)-\overline{g_{2}} Q\left(f_{1} v\right) \in \mathscr{D}_{h}$. By Lemma 7, we get that there is a $4 \times 4$ unitary matrix $U_{v}$ such that for some $1 \leq k \leq 5,\left\langle\left(f_{1}, Q\left(g_{1} v\right)\right.\right.$,
$\left.\left.g_{1},-Q\left(f_{1} v\right)\right), u_{j}\right\rangle_{C^{4}}$ are constants for $1 \leq j \leq k-1$ and $\left\langle\left(Q\left(\overline{g_{2}} v\right), \overline{f_{2}},-Q\left(\overline{f_{2}} v\right), \overline{g_{2}}\right), u_{j}\right\rangle_{C^{4}}$ are constants for $k \leq j \leq 4$.

Case 1. If there exists $v \in\left(\mathscr{D}_{h}\right)^{\perp}$ such that $k=1$ or $k=5$, it follows that $f_{1}, g_{1}$ are constants or $\overline{f_{2}}, \overline{g_{2}}$ are constants since $U$ is a unitary matrix. Hence we get that both $f$ and $g$ are holomorphic or both $\bar{f}$ and $\bar{g}$ are holomorphic.

Case 2. If there exists $v \in\left(\mathscr{D}_{h}\right)^{\perp}$ such that $k=2$ or $k=4$. We just prove the case of $k=4$; the case of $k=2$ is similar. Since $\left\langle\left(f_{1}, Q\left(g_{1} v\right), g_{1},-Q\left(f_{1} v\right)\right), u_{j}\right\rangle_{C^{4}}$ are constants for $1 \leq j \leq 3$, it follows that there exist a nonzero constant $t_{1}$ and a constant $c_{1}$ such that

$$
f_{1}(z)=t_{1} g_{1}(z)+c_{1} .
$$

Then by (46), we get

$$
\begin{aligned}
& (I-Q)\left[t_{1} g_{1} Q\left(\overline{g_{2}} v\right)+\overline{f_{2}} Q\left(g_{1} v\right)-g_{1} Q\left(\overline{f_{2}} v\right)\right. \\
& \left.-t_{1} \overline{g_{2}} Q\left(g_{1} v\right)\right]=0,
\end{aligned}
$$

which implies that

$$
g_{1}\left[t_{1} Q\left(\overline{g_{2}} v\right)-Q\left(\overline{f_{2}} v\right)\right]+Q\left(g_{1} v\right)\left[\overline{f_{2}}-t_{1} \overline{g_{2}}\right]
$$

is pluriharmonic. By Theorem 5.6 in [6], one of the following holds:

(1) Both $t_{1} Q\left(\overline{g_{2}} v\right)-Q\left(\overline{f_{2}} v\right)$ and $Q\left(g_{1} v\right)$ are constants.

(2) Both $t_{1} Q\left(\overline{g_{2}} v\right)-Q\left(\overline{f_{2}} v\right)$ and $\overline{f_{2}}-t_{1} \overline{g_{2}}$ are constants.

(3) There is a nonzero constant $t_{2}$ such that $Q\left(g_{1} v\right)-t_{2} g_{1}$ and $\left[t_{1} Q\left(\overline{g_{2}} v\right)-Q\left(\overline{f_{2}} v\right)\right]+t_{2}\left[\overline{f_{2}}-t_{1} \overline{g_{2}}\right]$ are constants.

If $\overline{f_{2}}-t_{1} \overline{g_{2}}$ is a constant, it follows easily that $f=t_{1} g+c$. Assume that $\overline{f_{2}}-t_{1} \overline{g_{2}}$ is not a constant. Then for all $v \in\left(\mathscr{D}_{h}\right)^{\perp}$, $g_{1}\left[t_{1} Q\left(\overline{g_{2}} v\right)-Q\left(\overline{f_{2}} v\right)\right]+Q\left(g_{1} v\right)\left[\overline{f_{2}}-t_{1} \overline{g_{2}}\right]$ is pluriharmonic if and only if one of the following holds:

(1) Both $t_{1} Q\left(\overline{g_{2}} v\right)-Q\left(\overline{f_{2}} v\right)$ and $Q\left(g_{1} v\right)$ are constants.

(2) There is a nonzero constant $t_{2}$ such that $Q\left(g_{1} v\right)-t_{2} g_{1}$ and $\left[t_{1} Q\left(\overline{g_{2}} v\right)-Q\left(\overline{f_{2}} v\right)\right]+t_{2}\left[\overline{f_{2}}-t_{1} \overline{g_{2}}\right]$ are constants.

Since $g_{1}$ is not a constant, similar to the previous proof, we can find $v_{\alpha^{\prime}} \in\left(\mathscr{D}_{h}\right)^{\perp}$ such that neither $Q\left(g_{1} v\right)$ nor $\mathrm{Q}\left(g_{1} v\right)-$ $t_{2} g_{1}$ is constant, which is a contradiction. Hence we get that $f=t_{1} g+c$.

Case 3. For all $v \in\left(\mathscr{D}_{h}\right)^{\perp}$, we have $k=3$. For each $v$, there exist constants $t_{1}, t_{2}$ and $c_{1}$ such that

$$
Q\left(f_{1} v\right)=t_{1} f_{1}+t_{2} g_{1}+c_{1} \text {. }
$$

Suppose that $f_{1}=\sum a_{m} z^{m}$ and $g_{1}=\sum b_{m} z^{m}$. For multi-index $\alpha$, let $v=z^{\alpha+d_{1}} \bar{z}^{d_{1}}-\left(\left(\alpha_{1}+1\right) /(n+|\alpha|)\right) z^{\alpha}$; there exists a holomorphic function $h$ such that $Q\left(f_{1} v\right)=z^{\alpha} h$. Then for all multi-index $m<\alpha$, we get $t_{1} a_{m}+t_{2} b_{m}=0$.

Note that $f_{1}$ and $g_{1}$ are not constants. If for every multiindex $m, a_{m} b_{m}=0$. Suppose that $a_{m^{1}} \neq 0$ and $b_{m^{2}} \neq 0$, where 
$m^{1} \neq m^{2}$. For any multi-index $\alpha$ satisfying $\alpha>m^{1}$ and $\alpha>$ $m^{2}$, let $v_{\alpha}=z^{\alpha+d_{1}} \bar{z}^{d_{1}}-\left(\left(\alpha_{1}+1\right) /(n+|\alpha|)\right) z^{\alpha}$. Then there exist constants $t_{1, \alpha}, t_{2, \alpha}$ and $c_{\alpha}$ such that

$$
Q\left(f_{1} v_{\alpha}\right)=t_{1, \alpha} f_{1}+t_{2, \alpha} g_{1}+c_{\alpha}
$$

From the above computation, for all nonzero $m<\alpha$, we have $t_{1, \alpha} a_{m}+t_{2, \alpha} b_{m}=0$. It follows that $t_{1, \alpha}=t_{2, \alpha}=0$. Then $Q\left(f_{1} v_{\alpha}\right)=c_{\alpha}$ for all $v_{\alpha}$ with $\alpha>m^{1}$ and $\alpha>m^{2}$. A direct computation gives

$$
\begin{aligned}
Q\left(f_{1} v_{\alpha}\right) & =z^{\alpha} \sum_{m \geq 0} a_{m}\left[\frac{m_{1}+\alpha_{1}+1}{n+|m|+|\alpha|}-\frac{\alpha_{1}+1}{n+|\alpha|}\right] z^{m} \\
& =z^{\alpha} \sum_{m \geq 0} a_{m} \frac{\alpha_{1}(n+|\alpha|)-|m|\left(\alpha_{1}+1\right)}{(n+|m|+|\alpha|)(n+|\alpha|)} z^{m} .
\end{aligned}
$$

It follows that, for all multi-index $m$, we have $a_{m}\left(\alpha_{1}(n+|\alpha|)-\right.$ $\left.|m|\left(\alpha_{1}+1\right)\right) /(n+|m|+|\alpha|)(n+|\alpha|)=0$. For each $m$, we can find a $v_{\alpha}$ such that $\alpha_{1}(n+|\alpha|)-|m|\left(\alpha_{1}+1\right) \neq 0$. Hence $a_{m}=0$ for all nonzero multi-index $m$. Then $f_{1}$ is a constant and this leads a contradiction.

Then there exists a multi-index $m_{0}$ such that $a_{m_{0}} \neq 0$ and $b_{m_{0}} \neq 0$. For any $\alpha>m_{0}$, let $v_{\alpha}=z^{\alpha+d_{1}} \bar{z}^{d_{1}}-\left(\left(\alpha_{1}+1\right) /(n+\right.$ $|\alpha|)) z^{\alpha}$. Similarly, we still have $t_{1, \alpha} a_{m_{0}}+t_{2, \alpha} b_{m_{0}}=0$. If $t_{1, \alpha}=0$, we get $t_{2, \alpha}=0$. Fix a multi-index $\beta>m_{0}$, suppose that, for all $v_{\alpha}$ with $\alpha>\beta$, we have $t_{1, \alpha}=t_{2, \alpha}=0$. It follows that $Q\left(f_{1} v_{\alpha}\right)=c_{\alpha}$ for all $\alpha>\beta$, which implies that $f_{1}$ is a constant. That is a contradiction. Suppose that there exists a multi-index $\beta^{1}>m_{0}$ such that $t_{1, \beta^{1}} \neq 0$. It follows that $t_{2, \beta^{1}} \neq 0$ and, for all $m<\beta^{1}, t_{1, \beta^{1}} a_{m}+t_{2, \beta^{1}} b_{m}=0$. If $a_{m}=0$, it follows that $b_{m}=0$. If $a_{m} \neq 0$, we get $b_{m}=-\left(t_{1, \beta^{1}} / t_{2, \beta^{1}}\right) a_{m}$. We also can find a multi-index $\beta^{2}$ with $\beta^{2}>\beta^{1}$ such that $t_{1, \beta^{2}} \neq 0$. Similarly, for all $m<\beta^{2}$, if $a_{m}=0$, we have $b_{m}=0$. If $a_{m} \neq 0$, we get $b_{m}=-\left(t_{1, \beta^{2}} / t_{2, \beta^{2}}\right) a_{m}$. Clearly, $-t_{1, \beta^{1}} / t_{2, \beta^{1}}=-t_{1, \beta^{2}} / t_{2, \beta^{2}}$. For each nonzero multi-index $m$, we can find a $v_{\beta}$ such that $b_{m}=t a_{m}$, where $t$ is a nonzero constant. Then we have $f_{1}=t g_{1}+c$, and similar to the proof of Case 2, we get that $f=\operatorname{tg}+c$.

By Lemma 5, the converse is easy to see. The proof is complete.

\section{Conflict of Interests}

The authors declare that there is no conflict of interests regarding the publication of this paper.

\section{Acknowledgment}

This research is supported by NSFC (nos. 11271059, 11271332, 11431011, and 11301047) and NSF of Zhejiang Province (nos. LY14A010013 and LY14A010021).

\section{References}

[1] A. Brown and P. R. Halmos, "Algebraic properties of Toeplitz operators," Journal für die Reine und Angewandte Mathematik, vol. 213, pp. 89-102, 1963-1964.
[2] S. Axler and Z. Čučković, "Commuting Toeplitz operators with harmonic symbols," Integral Equations and Operator Theory, vol. 14, no. 1, pp. 1-12, 1991.

[3] K. Stroethoff, "Essentially commuting Toeplitz operators with harmonic symbols," Canadian Journal of Mathematics, vol. 45, no. 5, pp. 1080-1093, 1993.

[4] S. Axler, Z. Čučković, and N. V. Rao, "Commutants of analytic Toeplitz operators on the Bergman space," Proceedings of the American Mathematical Society, vol. 128, no. 7, pp. 1951-1953, 2000.

[5] Z. Čučković and N. V. Rao, "Mellin transform, monomial symbols, and commuting Toeplitz operators," Journal of Functional Analysis, vol. 154, no. 1, pp. 195-214, 1998.

[6] D. Zheng, "Commuting Toeplitz operators with pluriharmonic symbols," Transactions of the American Mathematical Society, vol. 350, no. 4, pp. 1595-1618, 1998.

[7] B. R. Choe and Y. J. Lee, "Pluriharmonic symbols of commuting Toeplitz operators," Illinois Journal of Mathematics, vol. 37, no. 3, pp. 424-436, 1993.

[8] B. R. Choe and Y. J. Lee, "Pluriharmonic symbols of essentially commuting Toeplitz operators," Illinois Journal of Mathematics, vol. 42, no. 2, pp. 280-293, 1998.

[9] Y. J. Lee, "Pluriharmonic symbols of commuting Toeplitz type operators on the weighted Bergman spaces," Canadian Mathematical Bulletin, vol. 41, no. 2, pp. 129-136, 1998.

[10] Y. Lu, "Commuting of Toeplitz operators on the Bergman spaces of the bidisc," Bulletin of the Australian Mathematical Society, vol. 66, no. 2, pp. 345-351, 2002.

[11] B. R. Choe, H. Koo, and Y. J. Lee, "Commuting Toeplitz operators on the polydisk," Transactions of the American Mathematical Society, vol. 356, no. 5, pp. 1727-1749, 2004.

[12] S. Ohno, "Toeplitz and Hankel operators on the harmonic Bergman space,” RIMS Kokyuroku, vol. 946, pp. 25-34, 1996.

[13] B. R. Choe and Y. J. Lee, "Commuting Toeplitz operators on the harmonic Bergman space," Michigan Mathematical Journal, vol. 46, no. 1, pp. 163-174, 1999.

[14] B. R. Choe and Y. J. Lee, "Commutants of analytic Toeplitz operators on the harmonic Bergman space," Integral Equations and Operator Theory, vol. 50, no. 4, pp. 559-564, 2004.

[15] B. R. Choe and K. S. Nam, "Note on commuting Toeplitz operators on the pluriharmonic Bergman space," Journal of the Korean Mathematical Society, vol. 43, no. 2, pp. 259-269, 2006.

[16] Y. J. Lee and K. Zhu, "Some differential and integral equations with applications to Toeplitz operators," Integral Equations and Operator Theory, vol. 44, no. 4, pp. 466-479, 2002.

[17] K. Stroethoff and D. Zheng, "Algebraic and spectral properties of dual Toeplitz operators," Transactions of the American Mathematical Society, vol. 354, no. 6, pp. 2495-2520, 2002.

[18] Y. Lu, "Commuting dual Toeplitz operators with pluriharmonic symbols," Journal of Mathematical Analysis and Applications, vol. 302, no. 1, pp. 149-156, 2005.

[19] Y. F. Lu and S. X. Shang, "Commuting dual Toeplitz operators on the polydisk," Acta Mathematica Sinica (English Series), vol. 23, no. 5, pp. 857-868, 2007.

[20] Y. F. Lu and J. Yang, "Commuting dual Toeplitz operators on weighted Bergman spaces of the unit ball," Acta Mathematica Sinica (English Series), vol. 27, no. 9, pp. 1725-1742, 2011.

[21] J. Yang and Y. Lu, "Commuting dual Toeplitz operators on the harmonic Bergman space," Science China Mathematics, vol. 58, no. 7, pp. 1461-1472, 2015. 
[22] T. Yu and S. Y. Wu, "Algebraic properties of dual Toeplitz operators on the orthogonal complement of the Dirichlet space," Acta Mathematica Sinica, vol. 24, no. 11, pp. 1843-1852, 2008.

[23] T. Yu and S. Y. Wu, "Commuting dual Toeplitz operators on the orthogonal complement of the Dirichlet space," Acta Mathematica Sinica (English Series), vol. 25, no. 2, pp. 245-252, 2009.

[24] T. Yu, "Operators on the orthogonal complement of the Dirichlet space," Journal of Mathematical Analysis and Applications, vol. 357, no. 1, pp. 300-306, 2009.

[25] W. Rudin, Function Theory on the Unit Ball of $C^{n}$, vol. 241 of Grundlehren der Mathematischen Wissenschaften, Springer, Berlin, Germany, 1980.

[26] B. R. Choe, H. Koo, and Y. J. Lee, "Toeplitz products with pluriharmonic symbols on the Hardy space over the ball," Journal of Mathematical Analysis and Applications, vol. 381, no. 1, pp. 365-382, 2011.

[27] R. A. Adams, Sobolev Spaces, Academic Press, New York, NY, USA, 1975.

[28] P. Ahern and Z. Ččković, "A theorem of Brown-Halmos type for Bergman space Toeplitz operators," Journal of Functional Analysis, vol. 187, no. 1, pp. 200-210, 2001. 


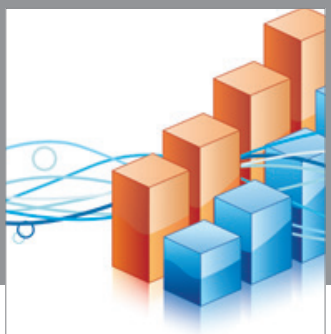

Advances in

Operations Research

vatem alat4

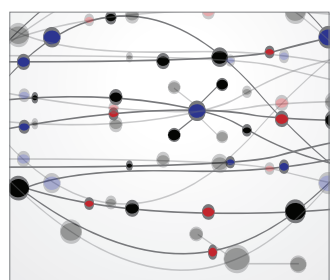

\section{The Scientific} World Journal
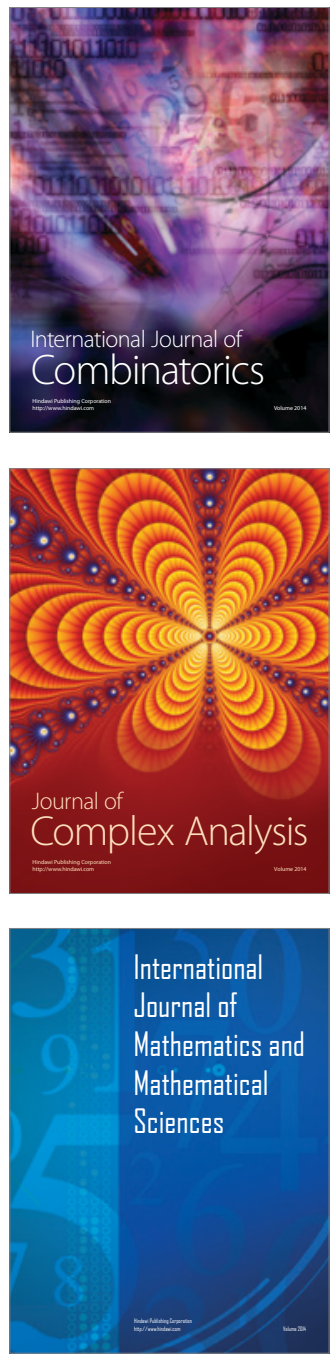
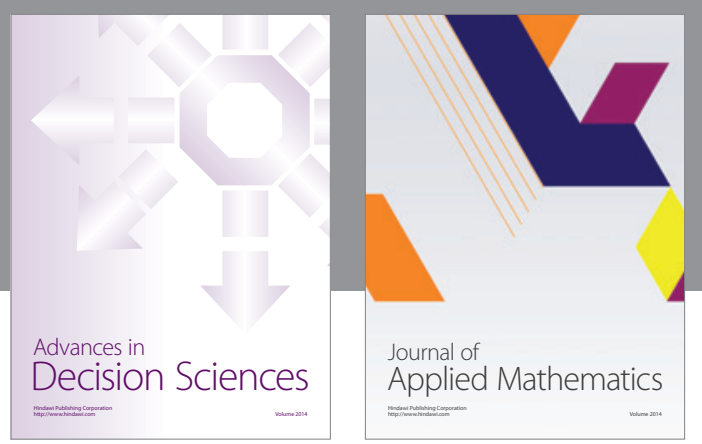

Algebra

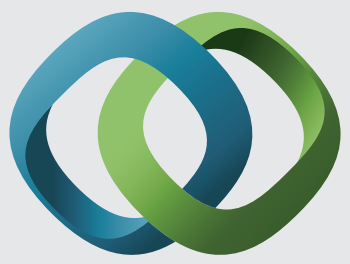

\section{Hindawi}

Submit your manuscripts at

http://www.hindawi.com
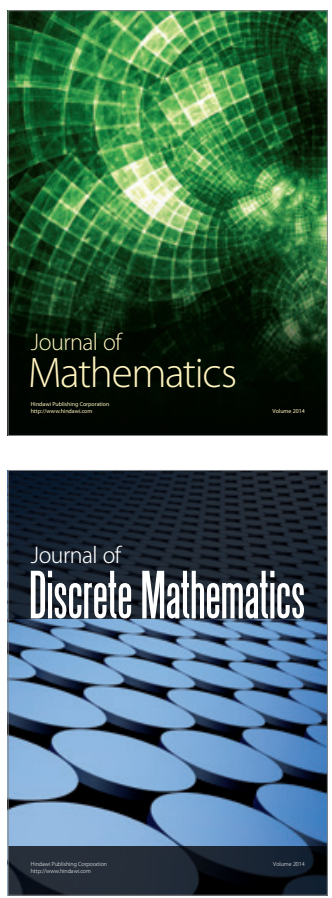

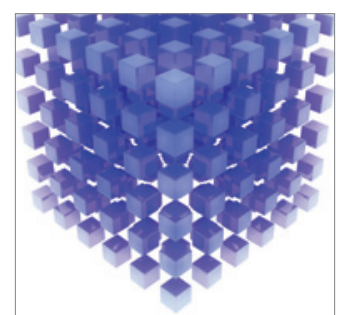

Mathematical Problems in Engineering
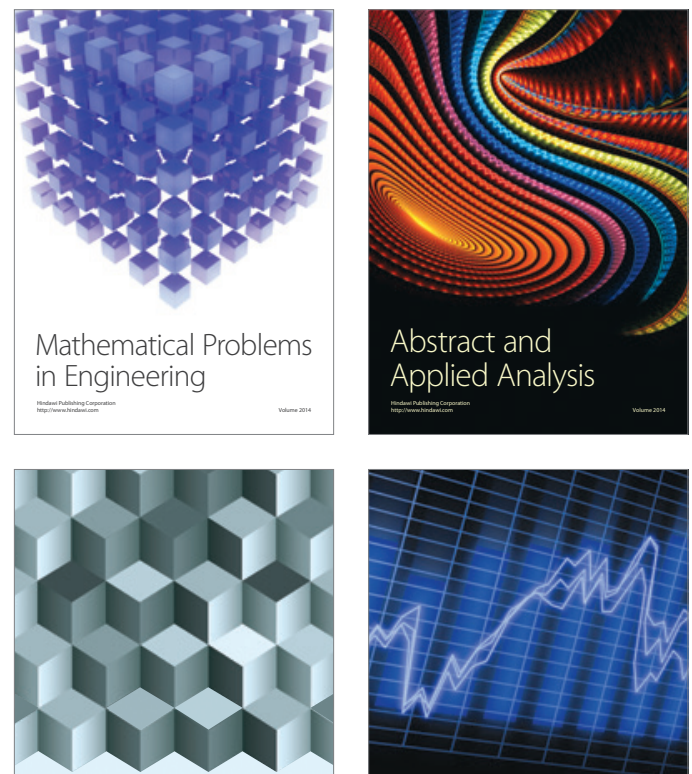

Journal of

Function Spaces

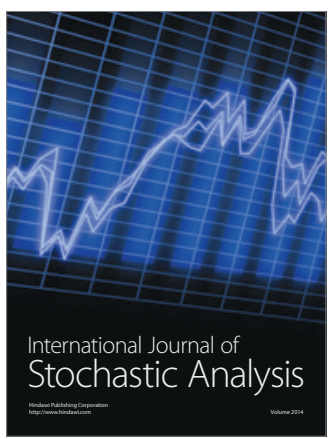

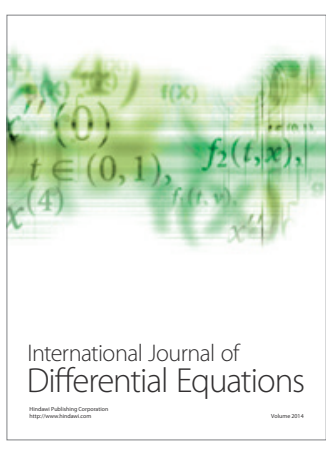
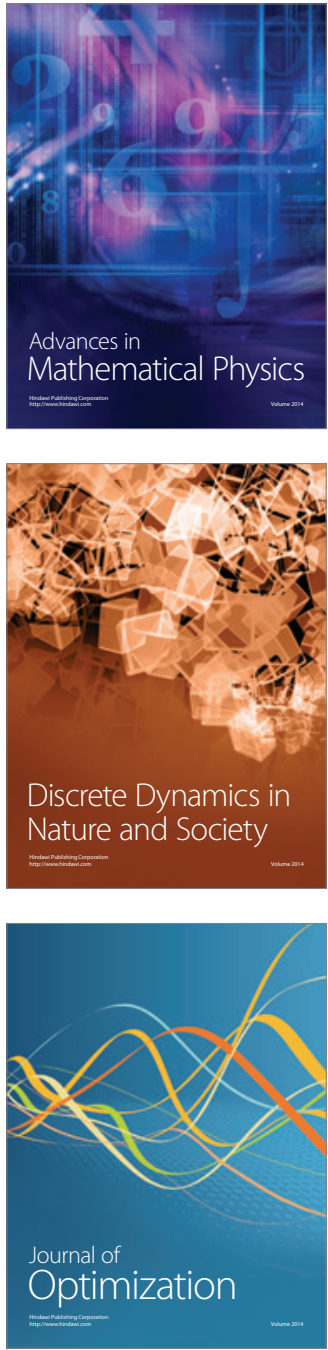